und Anatomie. Vielleicht greift ein Forscher dieser Disziplinen gelegentlich die Untersuchung der Abhängigkeit gewisser Details äußerer Körperformen von inneren Organen auf; vielleicht werden durch solche Untersuchungen dann auch die Längsfältchen und die Querfurche auf dem Halsschilde vieler Halticinen dem Verständnis näher gerückt.

\title{
Dritter Beitrag zum Vorkommen verschiedener paläarktischer Acalles-Arten (Col. Curc.).
}

\author{
Von Paul Meyer, Fiume. ${ }^{1}$ )
}

Von Herrn Gustav Pagan etti-Hummler, Vöslau, erwarb ich die Acalles, welche derselbe auf seinen verschiedenen Sammelreisen erbeutet hat, und benutze diese Gelegenheit, die interessanteren Fundorte nebst einigen anderen Beobachtungen hier bekanntzugeben.

A. denticollis Germ. Montepagano (östlich. Mittelitalien), Berge südlich von Castel di Sangro (südlich. Mittelitalien), Santa Eufemia (Kalabrien). An letzterem Platze kommen vollkommene Übergangsstücke zur sizilianischen Form minutesquamosus Reiche vor. Abschasien (Karl Rost).

A. camelus F. Monte Arazecca, Santa Eufemia.

A. Milleri Reitt. Abschasien (Karl Rost).

A. Aubei Boh. Monte Conero (Ancona), Monte Arazecca, Montepagano. Das Tier letzterer Provenienz wurde von Solari als Solarii Fiori bestimmt, welche Form sich von Aubei Boh. nicht spezifisch abtrennen läßt. Insel Elba (Paganetti 1908), Sotschi, Kaukasus (Karl Rost). Santa Eufemia (Kalabrien), Ponferrada (Asturien).

A. ptinoides Marsh. Asturien: Ponferrada, Caboalles, Cancas, Caril. A. roboris Curt. Elba, Garfagnana, Berge südlich von Castel di Sangro, Santa Eufemia.

A. pulchellus Bris. Elba.

A. caucasicus Reitt. Abschasien (Karl Rost).

A. hypocrita Boh. Berge südlich von Castel di Sangro, Montepagano. Ein zweifellos zu dieser Art gehöriges Tier aus Asturien (Cancas) trägt Solaris handschriftlichen Vermerk: „Ac. hispanicus nob.

1) Als ersten Beitrag bezeichne ich meine Notizen in dieser Zeitung vom Jahre 1908, S. 167-197, als zweiten Beitrag jene ebendaselbst, vom Jahre 1910, S. 28. Weitere Beiträge werden folgen. 
1912 var.". Nevesinje (Bosnien). Kaukasus (Karl Rost): Okum (Mingrelien), Bsyb (Abschasien), Kuban, Talysch.

A. variegatus Boh. Von seitens $\mathrm{Pag}$ an etti auf Korfu gesammelten 107 Acalles gehören 50 Exemplare zu variegatus Boh., 57 Exemplare zu Brisouti Reitt. Übergangstiere sind vorhanden. Brisouti Reitt. darf sicher nur als eine Form des variegatus Boh. angesehen werden. Mir liegen vor: A. variegatus Boh. Elba, Collesalvetti (bei Livorno), Topla (Karawanken-Kärnten), sehr kleine Tiere, Zelenika, Kameno, Sutorina, Kephallinia, die Form Brisouti Reitt.: Gerace und Santa Eufemia (Kalabrien), Berge südlich von Castel di Sangro, Montepagano, San Basilio (Murgien), Krivošije (Süddalmatien); die Form major Solari: Kri vošije, Zelenika.

A. validus Hampe. Ung.-Celic, Bosnien (Oskar Reiß).

A. lemur Germ. samt der Form Luigionii Solari: Monte Arazecca. Luigionii Sol. kann als selbständige Art nicht angesehen werden. A. turbatus Boh. samt der Form longus Desbr. (sehr kleine Stücke): Santa Eufemia, Kalabrien. Die Form echinatus Germ. sammelte Rost bei Sotschi (Kaukasus).

A. teter Boh. $\mathrm{Zu}$ dieser Art gehören vier von $\mathrm{Pag}$ a n etti in Asturien (Ponferrada und Cancas) gesammelte Käfer, welche einen handschriftlichen Vermerk Solaris tragen: "Ac. hispanicus nob. 1912".

A. Reinosae Bris. Asturien (Caboalles).

A. Diocletianus Germ. Süditalien, Murgien: San Basilio und Grottaglio; Kalabrien: Gerace. Algerien (Tarfaia), Théry. Die Tiere letzterer Provenienz gehören der Form aptus m. (uncatus Desbr.) an.

A. tuberculatus Rosenh. 1 Exemplar aus Asturien (Ponferrada), welches von Solari als zur portugiesischen Form lusitanicus gehörig bezeichnet wurde.

A. dromedarius Boh. Insel Elba (Paganetti 1908).

Das Auffinden des Ac. teter Boh. in Asturien ist sehr interessant, weil diese Art bisher aus Spanien nicht bekannt war. Der nächstverwandte $A c$. barbarus Luc. findet sich in Andalusien. Letzterer entwickelt sich an Disteln, am Erdboden, Ac. teter Boh. hingegen lebt auf Eichen. In Algier und Marokko kommen beide Arten vor. Daß die Exemplare des Ac. teter Boh. aus Asturien gegen jene 
von Korsika, Sardinien und Sizilien kleine Abweichungen aufweisen, berechtigt uns nicht, in den nordspanischen Tieren eine neue, selbständige Art zu erblicken !

Ich erinnere hier abermals an die bekannten schlesischen Exemplare des Ac. pyrenaeus Boh., für welche ich im Jahre 1895 schon eine Diagnose sub „Ac. Gerhardti m. nov. spec." entworfen hatte, als mich Reitter aufmerksam machte, daß sich dennoch die schlesischen Käfer von Tieren der Pyrenäen mit Recht spezifisch niemals abtrennen lassen würden. Ich bin Genanntem für jenen Wink noch heute dankbar und sehe seit langer Zeit schon die von mir im Jahre 1896 als spec. propr. beschriebenen drei Ac. globulus, affinis et aptus m. ebenfalls nur noch als Lokalrassen an, besser vielleicht noch, nur als ,Milieurassen“ (gemäß Dr. W. Ho r n, Dahlem). Ebenso die von Solari kreierten 16 Formen: Ac. Clermonti, denominandus, Ganglbaueri, Normandi, major, Pici, orientalis, Luigionii, dubius, squamosus, balcanicus, italicus, hispanicus, africanus, mauritanicus et lusitanicus Sol.

Für mich ist gerade die Gattung Acalles Schönh. aus dem Grunde so sehr interessant, weil beim kritischen Vergleiche eines entsprechend reichen Materiales der verschiedensten Gegenden, sich die morphologischen Unterschiede gerade so gut wie die äußerlichen und habituellen als nur anscheinend bestehend erweisen, da auch sie nicht konstant sind und sich durch Übergangsformen glatt überbrücken lassen.

Ein eingehendes Studium der paläarktischen Acalles zeigt sehr deutlich, wie die Natur auch bei den dieser Käfergattung angehörenden Formen sich in der Hypertelie, d. h. in der Hervorbringung einer nahezu endlosen Kette voneinander sich wohl im wesentlich Speziellen gleichenden, dennoch aber bald hier, bald dort sich unterscheidenden Individuen gefällt, welche, nach den Extremen auseinandergerissen und gliedweise ohne Zusammenhang einzeln betrachtet, den verschiedensten Autoren Anlaß zur Neubeschreibung von bereits benannten Arten gegeben haben. 


\section{$2 \mathrm{BHL}$ Biodiversity Heritage Library}

Meyer, Paul. 1915. "Dritter Beitrag zum Vorkommen verschiedener paläarktischer Acalles-Arten (Col. Curc.)." Wiener entomologische Zeitung 34, 396-398. https://doi.org/10.5962/bhl.part.10635.

View This Item Online: https://www.biodiversitylibrary.org/item/43833

DOI: https://doi.org/10.5962/bhl.part.10635

Permalink: https://www.biodiversitylibrary.org/partpdf/10635

\section{Holding Institution}

Smithsonian Libraries

\section{Sponsored by}

Smithsonian

\section{Copyright \& Reuse}

Copyright Status: NOT_IN_COPYRIGHT

This document was created from content at the Biodiversity Heritage Library, the world's largest open access digital library for biodiversity literature and archives. Visit BHL at https://www.biodiversitylibrary.org. 